\title{
Pien Tze Huang alleviate the joint inflammation in collagen-induced arthritis mice
}

\author{
YongQi Deng ${ }^{1,2}$, Hui Luo ${ }^{1,2}$, Jun Shu ${ }^{3}$, Haiyang Shu ${ }^{1,4}$, Cheng Lu' ${ }^{1}$ Ning Zhao ${ }^{1}$, Yun Geng ${ }^{2 *}$, Xiaojuan He ${ }^{1 *}$ \\ and Aiping Lu, $u^{5,6^{*}}$
}

\begin{abstract}
Background: Rheumatoid arthritis (RA) is an autoimmune disease characterized by synovitis. Pien Tze Huang (PZH) is a Chinese patent medicine with anti-inflammatory and immunomodulatory effects. However, whether PZH could be used in RA therapy is still unknown. Therefore, this study aimed to explore the therapeutic effect and the potential mechanism of PZH on collagen-induced arthritis (CIA) mice.

Methods: Male DBA/1J mice were used to establish an animal model of CIA and then treated with different doses of $\mathrm{PZH}$ for 4 weeks. The therapeutic effect of PZH on CIA mice was evaluated by arthritis score, pathological staining, and detecting the levels of inflammatory factors in serum and joints. To investigate its possible mechanism, the activity of NF-KB signaling pathway, NLRP3 inflammasome and the level of A20 were detected.

Results: The results showed that PZH could alleviate the erythema and swelling of hind paws of CIA mice, improve the pathological conditions of joint and decrease the production of IL-1 $\beta$, IL- 6 and IL-17 in serum and joints. Furthermore, PZH could significantly inhibit the activity of NF-KB signaling pathway and NLRP3 inflammasome in the ankle joint of CIA mice compared with the model group. It also increased the level of A20 in the ankle joint of CIA mice.
\end{abstract}

Conclusion: This study indicated that PZH could alleviate the joint inflammation of CIA mice, and the mechanism might be related to the regulation of NF-KB signaling pathway and NLRP3 inflammasome.

Keywords: Rheumatoid arthritis, Pien Tze Huang, NF-kB signaling pathway, NLRP3 inflammasome

\section{Background}

Rheumatoid arthritis (RA) is an inflammatory disease characterized by extensive synovial hyperplasia and inflammatory cell infiltration, which finally cause bone damage and functional joint disability [1]. Pro-inflammatory cytokines such as IL- $1 \beta$, IL- 6 and IL-17 produced by synoviocytes and infiltrating immune cells are highly

\footnotetext{
*Correspondence: jxgy88@126.com; hxj19@126.com; aipinglu@hkbu.edu.hk ${ }^{1}$ Institute of Basic Research in Clinical Medicine, China Academy of Chinese Medical Sciences, Beijing, China

${ }^{2}$ School of Life Science and Engineering, Southwest Jiaotong University, Chengdu, China

${ }^{5}$ Law Sau Fai Institute for Advancing Translational Medicine in Bone and Joint Diseases, School of Chinese Medicine, Hong Kong Baptist University, Kowloon Tong, Hong Kong, China

Full list of author information is available at the end of the article
}

associated with the pathogenesis of RA [2]. They could activate other inflammatory mediators like INF- $\gamma$, iNOS and COX-2, synergistically enhanced synovial inflammation, ultimately leading to joint damage [3]. Many signaling pathways have been proved to involve in regulating the production of pro-inflammatory cytokines in RA. Besides classical NF-kB signaling pathway, the NODlike receptor family pyrin domain-containing 3 (NLRP3) inflammasome in RA pathological progress gradually obtained concerns $[4,5]$. The activation of NLRP3 inflammasome influenced not only the expression of IL-1 $\beta$ and IL-18, but also the activation of NF- $\mathrm{KB}$ signaling pathway $[6,7]$. The transmission of these inflammatory signals also indicated the complexity of RA pathological process.

Clinical drugs for RA include non-steroidal antiinflammatory drugs (NSAIDs), glucocorticoids and

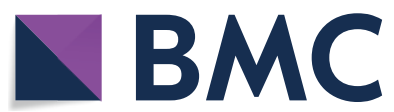

(c) The Author(s) 2020. This article is licensed under a Creative Commons Attribution 4.0 International License, which permits use, sharing, adaptation, distribution and reproduction in any medium or format, as long as you give appropriate credit to the original author(s) and the source, provide a link to the Creative Commons licence, and indicate if changes were made. The images or other third party material in this article are included in the article's Creative Commons licence, unless indicated otherwise in a credit line to the material. If material is not included in the article's Creative Commons licence and your intended use is not permitted by statutory regulation or exceeds the permitted use, you will need to obtain permission directly from the copyright holder. To view a copy of this licence, visit http://creativeco mmons.org/licenses/by/4.0/. The Creative Commons Public Domain Dedication waiver (http://creativecommons.org/publicdomain/ zero/1.0/) applies to the data made available in this article, unless otherwise stated in a credit line to the data. 
several biological agents, which have varying degrees of efficacy and side effects [8]. Therefore, safe and effective drugs are still urgently needed in RA treatment. Traditional Chinese medicines (TCM) are the treasure house for new drug discovery. Some TCM have been used to treat RA patients in clinic with good efficacy, such as WuTou Decoction and Yi Shen Juan Bi Pill $[9,10]$.

Pien Tze Huang (PZH), a well-known traditional Chinese formula, consists of Notoginseng Radix et Rhizoma (Tianqi or Sanqi), calculus bovis (Niuhuang or ox's gallstone), Shedan (snake's gall) and musk [11]. It has been used in China and Southeast Asia for centuries as a folk remedy for various inflammation related diseases, such as hepatitis. It was proved that PZH exerted anti-inflammatory effect by regulating the percentage of Th1 and Th17 cells, reducing the expression of IL-17, IL-23 and IFN- $\gamma$ and regulating NF- $\mathrm{kB}$ and STAT signaling pathways in inflammatory model animals [11, 12]. Recently, some medicinal components of Notoginseng Radix et Rhizoma have been reported to have remarkable effect in treating mice or rats with collagen-induced arthritis (CIA), a classical RA animal model $[13,14]$. In addition, ginsenoside Rg1 and ginsenoside metabolite compound $\mathrm{K}$ could markedly regulate NLRP3 inflammasome and NF- $\mathrm{kB}$ nuclear translocation $[14,15]$. Based on these studies, we hypothesized that PZH might have therapeutic effect on CIA mice. Therefore, this research aimed to explore the therapeutic potential and possible mechanism of PZH on CIA mice.

\section{Methods}

\section{Drugs}

PZH was produced by Zhangzhou Pien Tze Huang Pharmaceutical Co., Ltd., (Zhangzhou, China; FDA approval no. Z35020243). The drug samples were identified and characterized by HPLC-MS/TOF as our previous publication [12]. Stock solution of PZH was prepared by dissolving the $\mathrm{PZH}$ powder in purified water and the sample was fully blended again prior to use.

\begin{abstract}
Animals
Male 6-8 week old DBA/1J mice were obtained from Beijing Vital River Laboratories (Beijing, China). The mice were fed with food and water ad libitum, and then were allowed to acclimatize themselves for 1 week before the initiation of experiment. All protocols used here received approval from the Research Ethics Committee of Institute of Basic Theory of Chinese Medicine, China Academy of Chinese Medical Sciences.
\end{abstract}

\section{Induction of $\mathrm{CIA}$}

Bovine type II collagen (CII, $2 \mathrm{mg} / \mathrm{mL}$; Chondrex, Redmond, WA, USA) was diluted in an equal volume of complete Freund's adjuvant (Chondrex). Mice were first immunized with $100 \mu \mathrm{L}$ emulsion containing $100 \mu \mathrm{g}$ CII via intradermal injection into the tail and followed by a booster immunization on day 21 [16]. The onset of CIA could be observed between day 28 and day 32 after the first immunization.

\section{Treatment}

We divided the mice of successful induction of CIA model into six groups (six mice per group), which were normal group, model group, $\mathrm{PZH}$ low dose group (PZH$\mathrm{L}, 0.078 \mathrm{~g} / \mathrm{kg} /$ day), $\mathrm{PZH}$ middle dose group (PZH-M, $0.234 \mathrm{~g} / \mathrm{kg} /$ day, equal to the clinical dose), $\mathrm{PZH}$ high dose group (PZH-H, $0.702 \mathrm{~g} / \mathrm{kg} /$ day), and methotrexate group (MTX, $0.3 \mathrm{mg} / \mathrm{kg} /$ day, set as previously described [17]). Purified water (normal group, model group), MTX and PZH were intragastrically administered every day at a volume of $1 \mathrm{~mL} / 100 \mathrm{~g}$ for 4 weeks.

\section{Arthritis score}

The arthritis score was measured twice a week. Arthritis score for each ankle joint was recorded by the same observers, who were blind to the treatment received by animals as previously described. Scoring was performed with a $0-4$ scale, the arthritis scores were performed as: $0=$ no change; $1=$ paw with mild swelling at single limb; $2=$ more than one paw with swelling; $3=$ all paw with swelling and obvious erythema; and $4=$ whole paw with severe swelling. The maximum score of each mouse was $16[18]$.

\section{ELISA}

Samples were prepared from the mice of serum. The levels of IL-1 $\beta$, IL- 6 and IL-17 in serum of mice were determined by ELISA using commercial kits (Invitrogen, Carlsbad, CA, USA) according to the manufacturer's protocol.

\section{Blood biochemical determination}

Alanine aminotransferase (ALT), aspartate aminotransferase (AST), creatinine (CREA) and urea (UREA) in serum of mice were tested with blood biochemical commercial kits by a Japan's Hitachi 7160 automatic biochemical analyzer.

\section{Histopathology}

The sections $(3 \mu \mathrm{m})$ were prepared and stained with hematoxylin and eosin (H\&E). Histopathological characteristics were assessed and scored under blinded conditions according to the following system: 0 , no detectable change; 1 , minor focal infiltrate; 2 , moderate infiltrate; 3 , severe infiltrate but no pannus or erosion of cartilage; and 4 , very severe infiltrate plus pannus or cartilage erosion 
and fibrosis [19]. The scoring was performed blindly by three independent observers.

\section{Immunohistochemistry}

The sections were incubated respectively with anti-IL-1 $\beta$ (dilution 1:1000, Abcam, Cambridge, UK), anti-IL-6 (dilution 1:1000, Abcam, Cambridge, UK), anti-IL-17 (dilution 1:200, Abcam, Cambridge, UK) and anti-A20 (dilution 1:200, Abcam, Cambridge, UK) overnight at $4{ }^{\circ} \mathrm{C}$, followed by hatching with biotinylated horse radish peroxidase-conjugated secondary antibody for $30 \mathrm{~min}$ at room temperature (ZSGB-BIO, Beijing, China). Peroxidase staining was developed with DAB Kit (Cell Signaling Technology, Danvers, MA, USA). Later, sections were subjected to counterstain with hematoxylin (Leagene, Beijing, China). In the negative control groups, PBS buffer was used instead of the primary antibodies. Images were captured by LEICA DM6000B with LEICA DFC300 FX at a magnification of $400 \times$. Semi-quantitative analysis was performed as previously described [20].

\section{Western blotting}

The ankle joints were lysed by RIPA Lysis Buffer (Beyotime, Shanghai, China), which involved PMSF (Beyotime, Shanghai, China). According to the manufacturer's instructions, protein samples in RIPA buffer were quantified with the Micro BCA protein assay kit (Beyotime, Shanghai, China). Each sample was used for electrophoresis with $10 \%$ sodium dodecyl sulfate-polyacrylamide gels and then transferred to PVDF membranes. The PVDF membranes were blocked with $3 \%$ bovine serum albumin in Tris buffered saline-Tween 20 (TBST) buffer for $30 \mathrm{~min}$ at room temperature. The blots were incu-

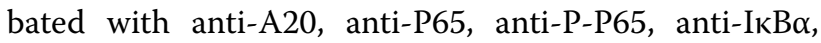
anti-NLRP3, anti-ASC and anti-caspase-1 antibody at 1:1000 overnight at $4{ }^{\circ} \mathrm{C}$ and with horse radish peroxidase-conjugated secondary antibody (Abcam, Cambridge, UK) for $60 \mathrm{~min}$ at room temperature. Blots were scanned and analyzed with Image J software.

\section{Statistical analysis}

All of the data were analyzed using GraphPad Prism 6 software and were expressed as the mean \pm standard deviation (SD) using one-way ANOVA. Difference with $\mathrm{P}$-value $<0.05$ was considered significant.

\section{Results}

\section{$\mathrm{PZH}$ ameliorated the symptoms of $\mathrm{CIA}$ mice}

To investigate the effect of $\mathrm{PZH}$ on arthritis, different dose of PZH were orally administered to the CIA mice for 4 consecutive weeks, and MTX was used as a positive control. The erythema and swelling of the hind paws in CIA mice could be markedly observed, while PZH treatment ameliorated the arthritis symptoms, inhibited the swelling and erythema of the hind paws in CIA mice (Fig. 1a). Consistently, arthritis scores in PZHtreated CIA mice were significantly lower than those in model group (Fig. 1b). Histologic evaluation of the ankle joints revealed the inflammatory cells infiltration, synovial hyperplasia and joint destruction in model group. Whereas, PZH treatment could alleviate those histopathological changes (Fig. 1c, d).

\section{PZH decreased the production of cytokines in serum and joints of $\mathrm{CIA}$ mice}

To evaluate the anti-inflammatory effect of $\mathrm{PZH}$, the ELISA and immunohistochemistry analysis were used to detect the levels of pro-inflammatory cytokines in serum and synovium, respectively. The data indicated that the levels of IL-1 $\beta$, IL- 6 and IL-17 in serum (Fig. 2a-c) and synovium (Fig. 3a-c) of CIA mice were significantly increased in model group compared with the normal group, and decreased in groups treated with PZH compared with the model group.

\section{PZH inhibited the NF-KB signal pathway in the joints of ClA mice}

As the above results showed that PZH-M had better effect on CIA mice, we then chose PZH-M for further mechanism study. We firstly detected the expression of P65, P-P65 and IкB $\alpha$ in the ankle joints of mice by western blotting. As shown in Fig. 4, the level of P-P65 was significantly increased in model group compared with the normal group, and decreased in PZH treated group compared with the model group. By contrast, the level of $\mathrm{I} \kappa \mathrm{B} \alpha$ was decreased in model group compared with the normal group, and increased in PZH treated group compared with the model group. In addition, the level of P65 had no significant difference in all groups.

\section{PZH suppressed the NLRP3 inflammasome in the joints of CIA mice}

To investigate the anti-inflammatory mechanism of $\mathrm{PZH}$ in CIA mice, the expression of NLRP3, ASC and activated caspase-1 (caspase-1 P20) in the ankle joints of mice were detected by western blotting. The results showed that the levels of NLRP3, ASC and caspase-1 P20 was significantly increased in the model group compared with the normal group, whereas PZH treatment decreased the levels of these factors when compared to the model group (Fig. 5).

\section{$\mathrm{PZH}$ increased the production of $\mathrm{A} 20$ protein in the joints of CIA mice}

To further explore whether the anti-inflammatory mechanism of $\mathrm{PZH}$ was relative to the expression of A20, the western blotting and immunohistochemistry analysis 
a

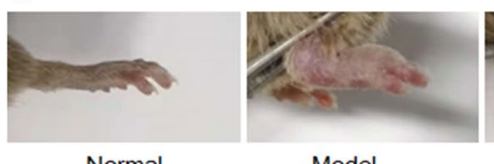

Normal

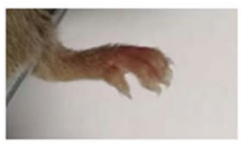

$\mathrm{PZH}-\mathrm{L}$

c
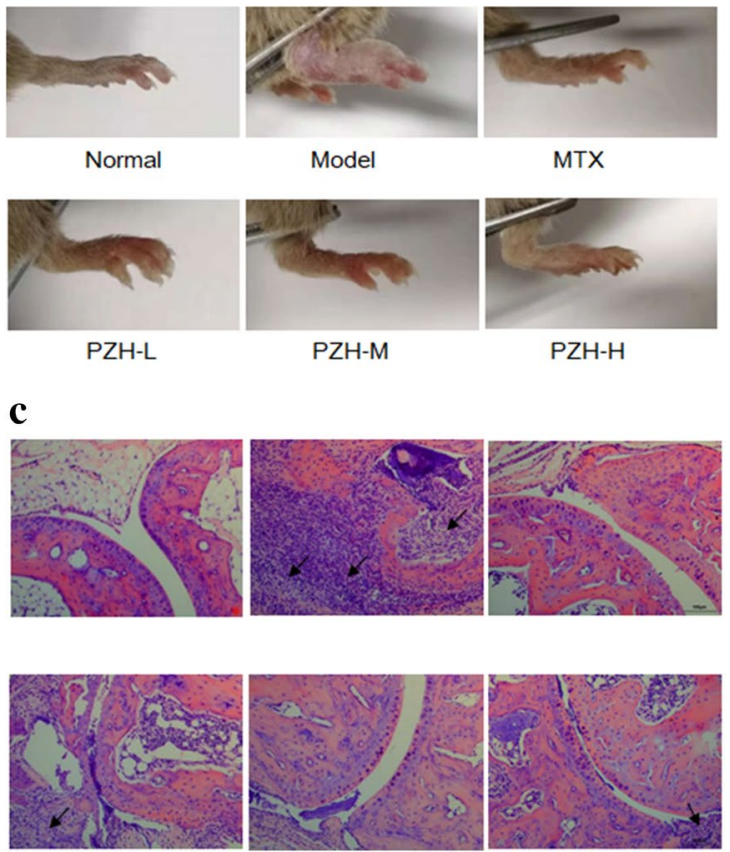

$\mathrm{PZH}-\mathrm{H}$ b

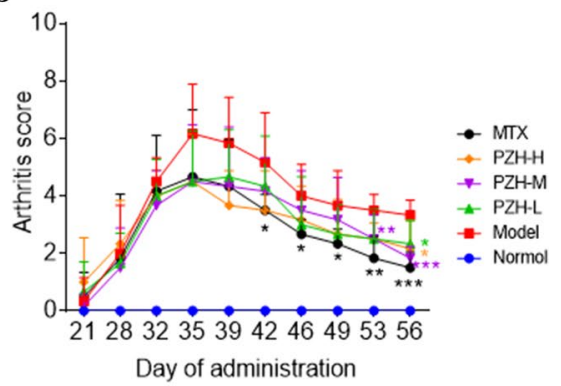

d

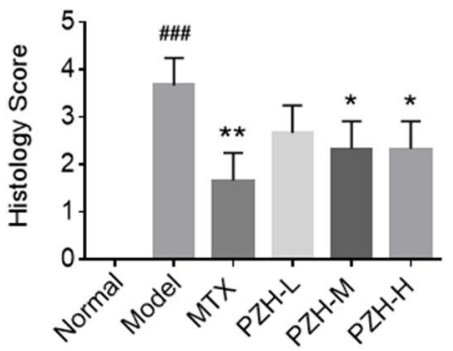

Fig. $1 \mathrm{PZH}$ ameliorated the symptoms of ClA mice. Mice were orally administered with PZH-L (0.078 g/kg/day), PZH-M (0.234 g/kg/day), PZH-H $(0.702 \mathrm{~g} / \mathrm{kg} /$ day), MTX $(0.3 \mathrm{mg} / \mathrm{kg} /$ day) or pure water (both Normal group and Model group) for 28 days. a Representative photograph of ankle joint from each group. b Arthritis score of each group. c Representative pathological sections of the ankle joint by H\&E staining ( $\times 200$, scale bars $=100 \mu \mathrm{m})$. The black arrow indicates the inflammatory site. $\mathbf{d}$ Histology score of each group. $n=6$ each group. ${ }^{\# \#} \mathrm{P}<0.001, \mathrm{vs}$. normal group, ${ }^{*} \mathrm{P}<0.05,{ }^{* *} \mathrm{P}<0.01,{ }^{* *} \mathrm{P}<0.001$, vs. model group
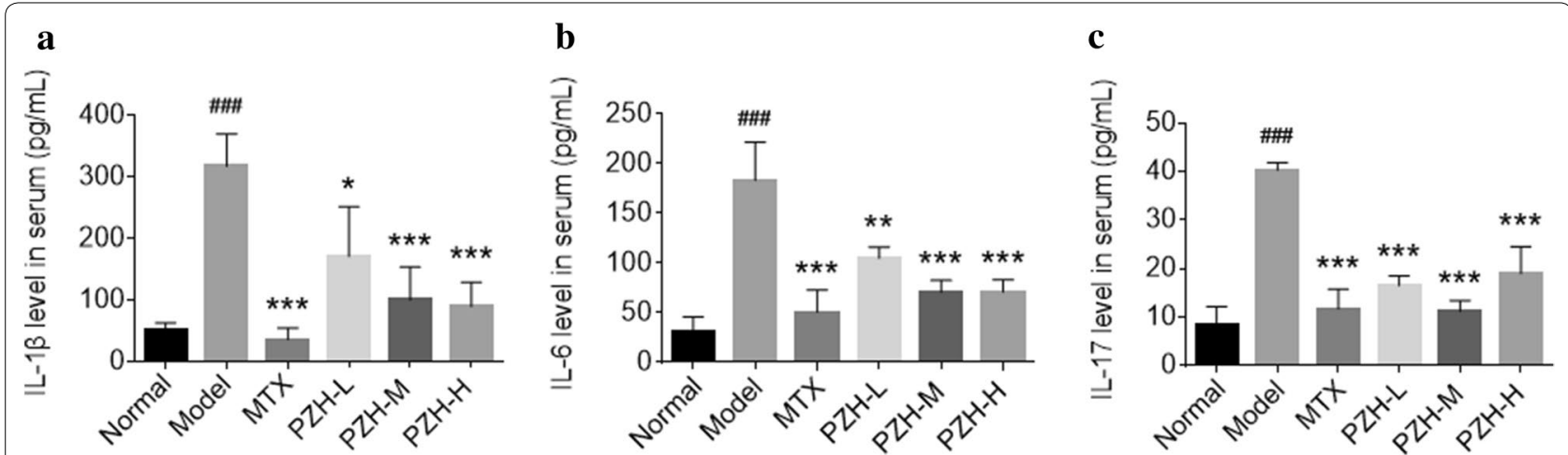

Fig. 2 PZH suppressed the production of pro-inflammatory cytokines in serum of CIA mice. The levels of IL-1 $\beta$ a, IL- $6 \mathbf{b}$ and IL-17 $\mathbf{c}$ in serum of mice were determined by ELISA. $n=6$ each group. ${ }^{\# \#} P<0.001$, vs. normal group, ${ }^{*} P<0.05$, ${ }^{* *} P<0.01$, ${ }^{* * *} P<0.001$, vs. model group

were used to detect the level of the protein in the ankle joints and synovium of mice, respectively. As shown in Fig. 6, in the model group, the level of A20 significantly decreased when compared with the normal group. $\mathrm{PZH}$ treatment significantly increased the production of A20 when compared with the model group.

\section{$\mathrm{PZH}$ had no significant toxicity in CIA mice}

To investigate whether PZH had toxicity in CIA mice, the levels of ALT and AST for hepatotoxicity as well as CREA and UREA for nephrotoxicity were detected. As shown in Fig. 7, PZH treatment didn't significantly change the levels of ALT, AST, CREA and UREA. 


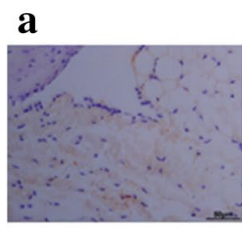

Normal

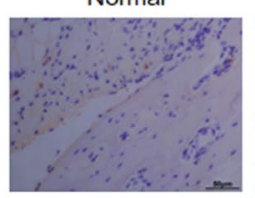

$\mathrm{PZH}-\mathrm{L}$

b

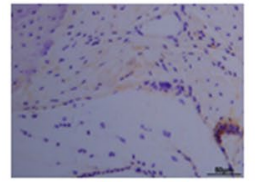

Normal

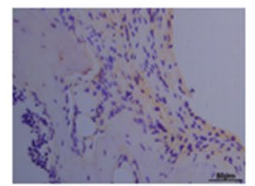

PZH-L

c

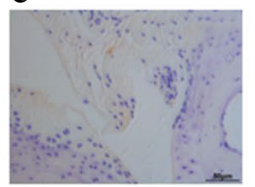

Normal

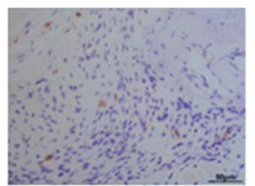

$\mathrm{PZH}-\mathrm{L}$

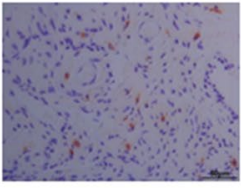

Model

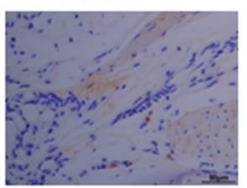

$\mathrm{PZH}-\mathrm{M}$

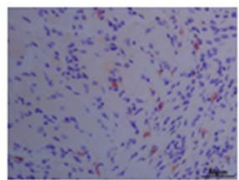

Model

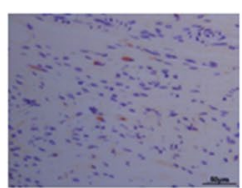

$\mathrm{PZH}-\mathrm{M}$

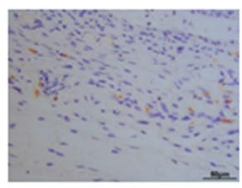

Model

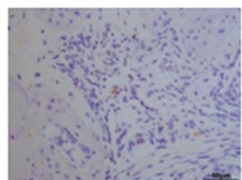

$\mathrm{PZH}-\mathrm{M}$

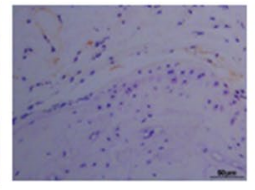

MTX

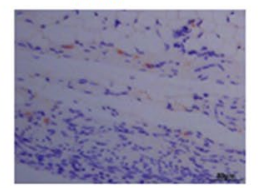

$\mathrm{PZH}-\mathrm{H}$

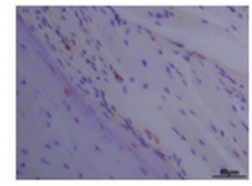

MTX

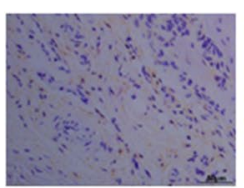

$\mathrm{PZH}-\mathrm{H}$

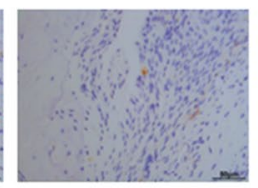

MTX

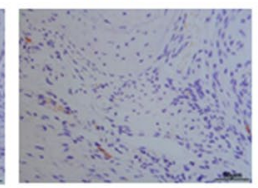

$\mathrm{PZH}-\mathrm{H}$

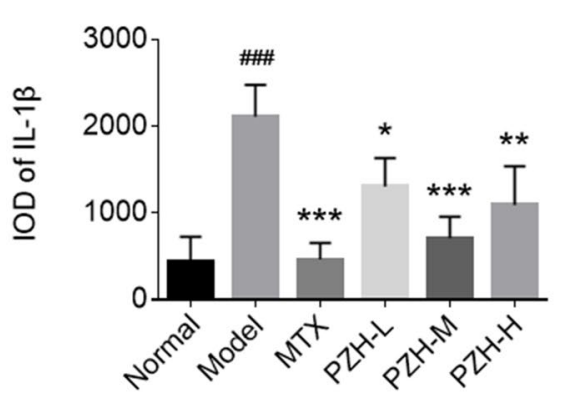

Fig. $3 \mathrm{PZH}$ decreased the levels of pro-inflammatory cytokines in the joint of CIA mice. Representative immunohistochemistry images (left) and

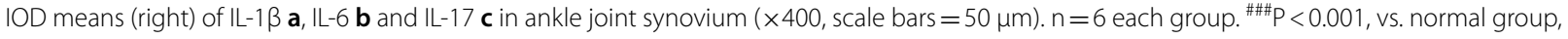
${ }^{*} \mathrm{P}<0.05$, ${ }^{* *} \mathrm{P}<0.01,{ }^{* * *} \mathrm{P}<0.001$, vs. model group

\section{Discussion}

PZH is an anti-inflammatory Chinese patent medicine, having therapeutic effect on hepatitis, cholecystitis and other diseases. Recently, PZH was reported to alleviate some inflammatory autoimmune diseases such as multiple sclerosis [11]. However, whether PZH could be used to treat RA was still uncertain. In this study, we immunized DBA/1J mice with bovine type II collagen to induce a CIA model and treated these CIA mice with different doses of PZH. We found that PZH could significantly relieve the erythema and swelling of the hind paws, and improve the pathological conditions in the ankle joints of CIA mice. The inflammatory cells infiltration, synovial inflammation and joint damage in CIA mice were alleviated after treatment with PZH. These results implied that PZH could be used to treat RA. Simultaneously, blood biochemical determination indicated that PZH did not show significant liver and kidney functional impairment.

The main pathological phenomenon of RA was synovitis. Long-term inflammation of synovium would accelerate the lesion and eventually aggravate joint dysfunction and malformation. Synovitis was caused by persistent inflammatory responses, which were resulted from the increased number of inflammatory cells and 

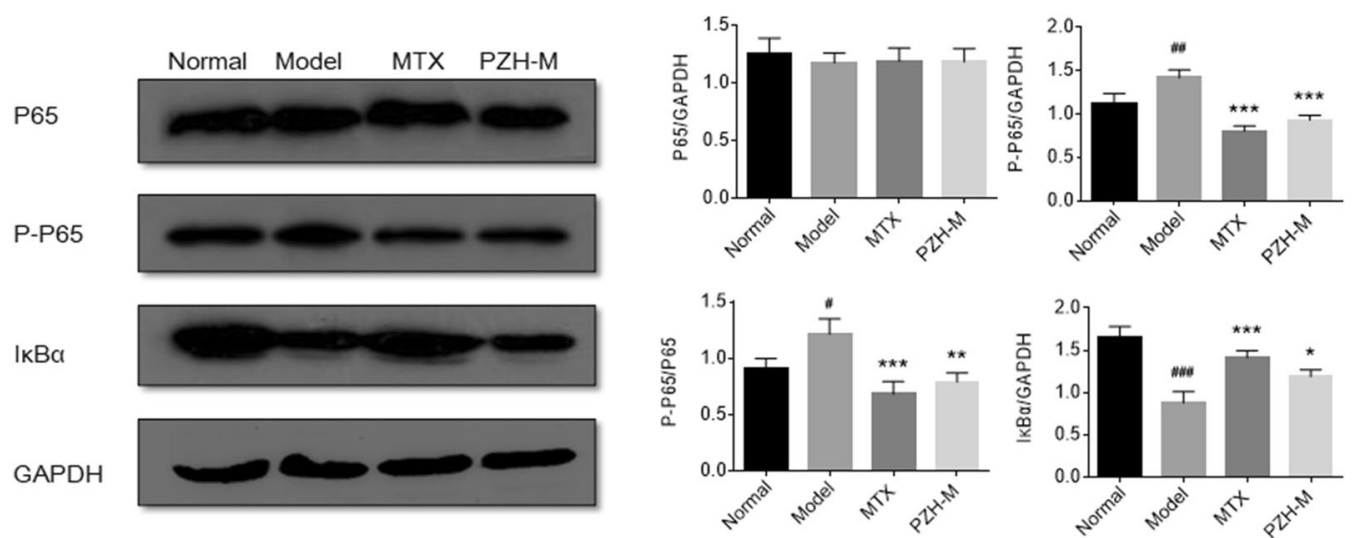

Fig. $4 \mathrm{PZH}$ inhibited NF-KB signal pathway in the joints of CIA mice. Western blot bands of P65, P-P65 and IkBa (left) and their expression relative to GAPDH or P65 (right). All experiments were performed three times. ${ }^{\# \#} \mathrm{P}<0.01,{ }^{\# \# \#} \mathrm{P}<0.001$, vs. normal group, ${ }^{*} \mathrm{P}<0.05$, ${ }^{* *} \mathrm{P}<0.01$, ${ }^{* * *} \mathrm{P}<0.001$ vs. model group

the increased secretion of various inflammatory mediasynovium to observe the effect of PZH on CIA mice.

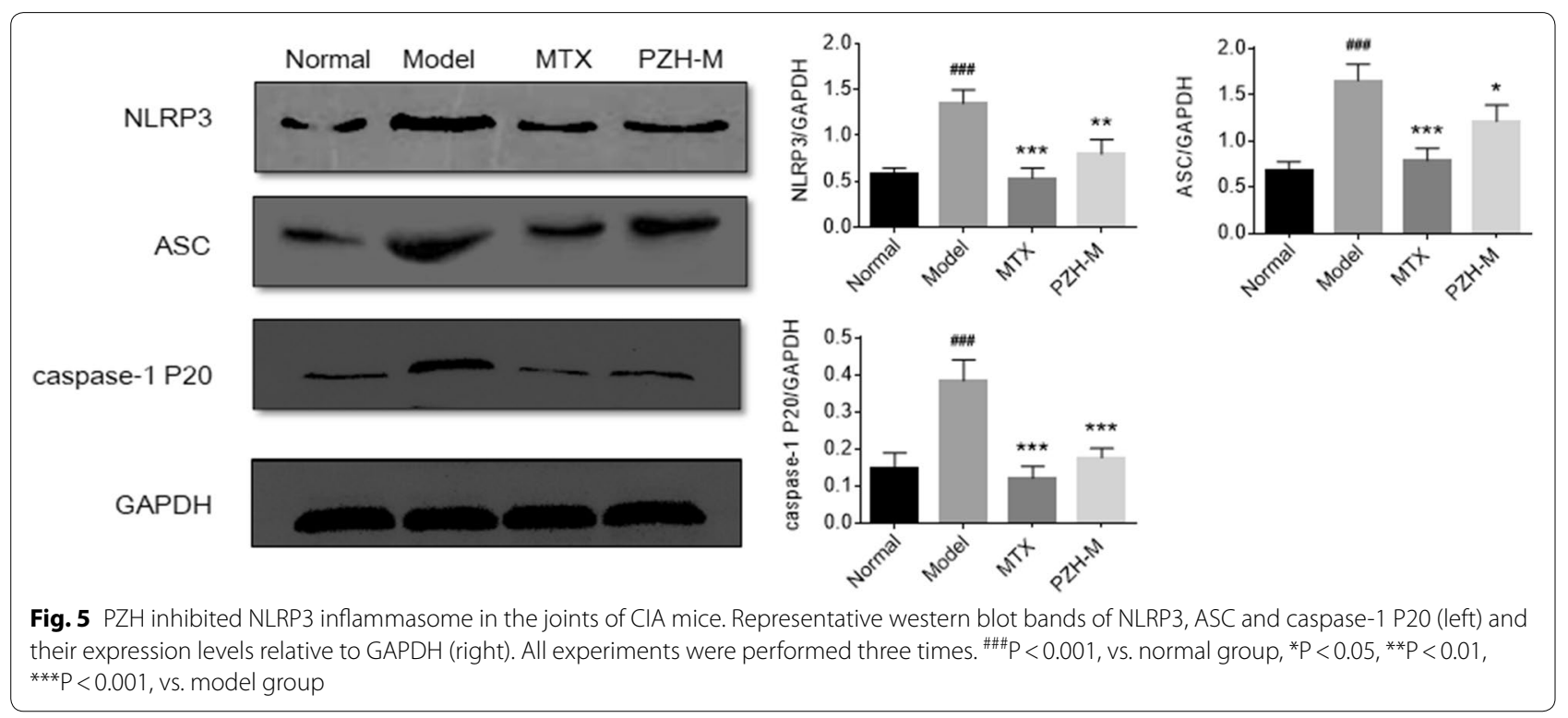

tors, such as IL-1 $\beta$, IL-6 and IL-17. These cytokines could make $\mathrm{T}$ cells, B cells, mononuclear cells and neutrophils collect in the diseased joints, and make synoviocytes proliferation and fibrosis, stimulate synoviocytes to produce more colony stimulating factor, chemokines, proinflammatory factors and other inflammatory mediators to amplify the inflammatory response [21-23]. Currently, some inflammatory factor inhibitors have been clinically used to treat RA, including IL-6 inhibitor tocilizumab and IL-1 $\beta$ inhibitor anakinra $[24,25]$. In this study, we detected the levels of IL-1 $\beta$, IL-6, IL-17 in serum and
We found that PZH could inhibit the expression of proinflammatory factors IL-1 $\beta$, IL- 6 and IL-17 in CIA mice, which also reflected the therapeutic effect of $\mathrm{PZH}$ on CIA mice.

Inflammatory cytokines expressed in a variety of cells and regulated by signaling pathways, which played important roles in mediation of immune and inflammatory responses in RA [26]. The most classical signal transduction pathways in RA included NF-kB signal pathway $[27,28]$. Stimulation of inflammatory signals caused the continued activation of the NF- $\kappa B$ pathway, followed by 


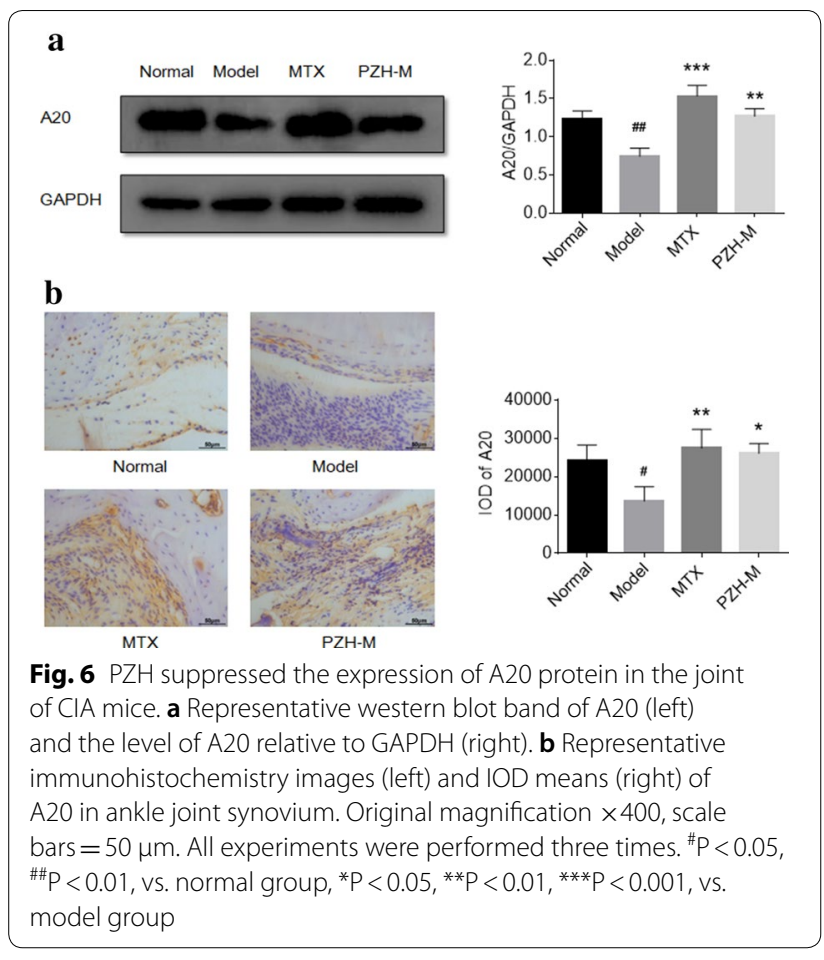

inhibitory protein IкB $\alpha$ degraded, resulting to release P65 [29]. After phosphorylation, the active molecules P-P65 bound to the promoter regions of different genes, causing abnormal expressions of downstream inflammatory factors including IL-1 $\beta$, IL-6 and IL-17 [30]. Previous studies have reported that the effective component of PZH, ginsenoside $\mathrm{Rf}, \mathrm{Rd}, \mathrm{Rh} 2$ and notoginsenoside $\mathrm{R} 1$ could regulate the activity of NF- $\mathrm{KB}$ signal pathway [31-34]. Our experimental results showed that PZH could inhibit the activity of NF- $\kappa B$ signal pathway in the ankle tissue of CIA mice, which implied that the anti-inflammatory effect of PZH might be related to the regulation of NF- $\kappa \mathrm{B}$ signal pathway.

The NLRP3 inflammasome is an intracellular multimolecular complex that controls caspase- 1 activity in response to various pathogen-derived factors as well as danger-associated molecules [35]. Recently, some studies indicated that NLRP3 inflammasome was involved in the pathogenesis of RA $[4,36]$. The activation of NLRP3 inflammasome could be induced by toll-like receptor (TLR) and NF-kB pathway [37]. NLRP3 recruited and activated pro-caspase-1 through ASC protein, the pro-caspase- 1 was converted into caspase- 1 and then
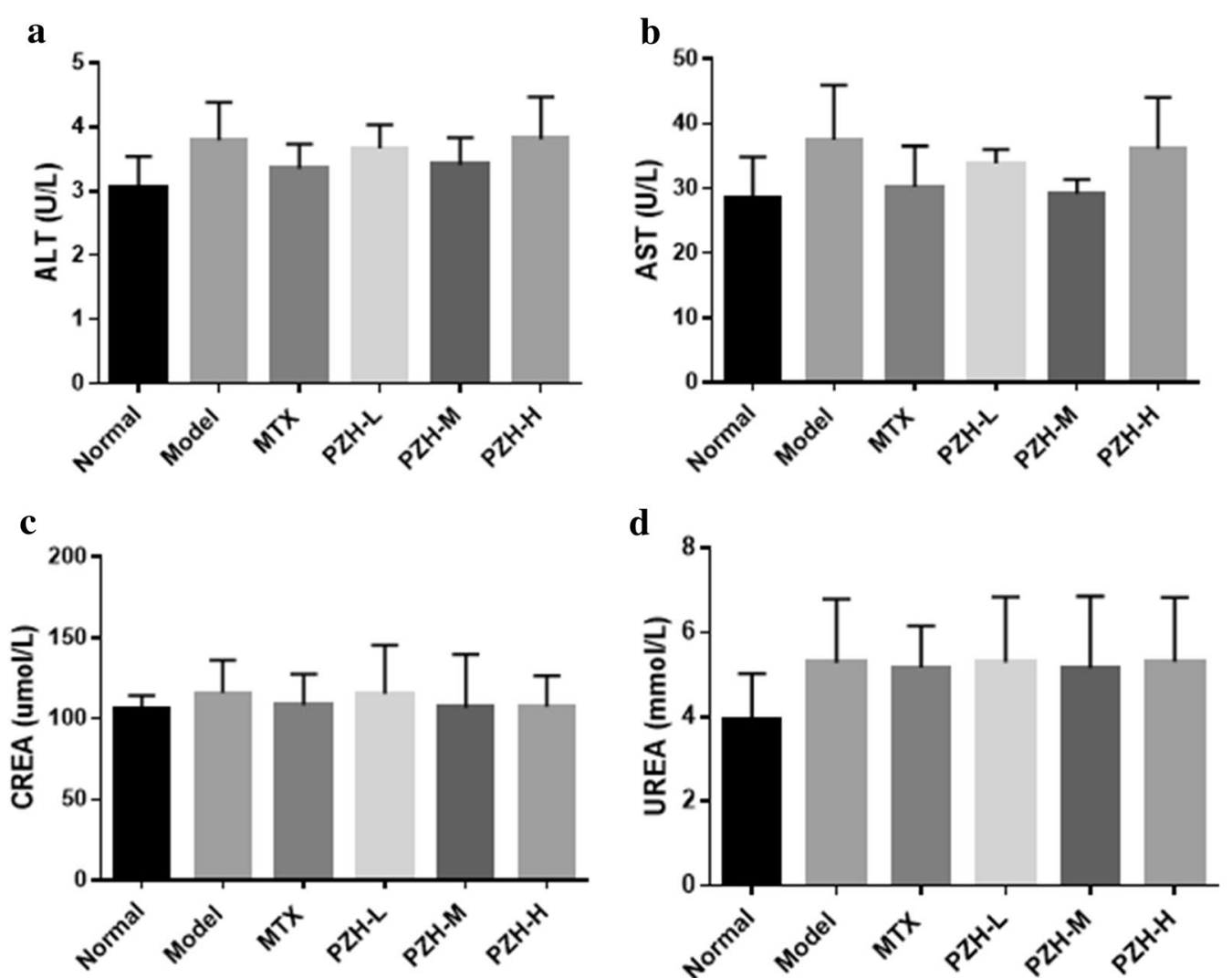

Fig. 7 PZH had no significant toxicity in CIA mice. The levels of ALT $\mathbf{a}$, AST $\mathbf{b}, C R E A \mathbf{c}$ and UREA $\mathbf{d}$ in serum of mice were shown respectively. $n=6$ each group 
formed inflammasome, which activated pro-IL-1 $\beta$ and pro- IL-18 to release large amounts of IL- $1 \beta$ and IL-18 [4]. Stimulation of IL-1 $\beta$ caused the continuous activation of the NF- $\mathrm{kB}$ pathway and secretion of inflammatory cytokines directly or indirectly. These inflammatory cytokines could make NLRP3 inflammasome highly activated and expressed more IL-1 $\beta$ and IL-18, with persistent synovium inflammation and bone degradation of the joints, leading to severe joint lesions and even loss of motor function. It was reported that the effective components of PZH, ginsenoside $\operatorname{Rg} 1[14,38]$ and ginsenoside metabolite compound $\mathrm{K}$ [15], had an inhibitory effect on the activity of NLRP3 inflammasome. Our study further found that PZH could inhibit the activation of NLRP3 inflammasome in the joint of CIA mice.

Zinc finger protein A20, also known as tumor necrosis factor $\alpha$ induced protein 3 (TNFAIP3), is a key molecule that negatively regulates the inflammatory response $[39,40]$. A20 exerts biological effects through the editing function of ubiquitination, which is mainly reflected in the regulation of cell differentiation and apoptosis, inflammatory response and immune regulation [41]. Previous studies indicated that $\mathrm{A} 20$ was an effective negative regulator of NLRP3 inflammasome and NF- $\mathrm{kB}$ signaling pathway [27, 42]. In RA patients, the expression of A20 in peripheral blood mononuclear cells and synovial fluid of joints was significantly lower than normal level [43]. By using CIA mice with A20 knockout, a research revealed that A20 could inhibit the activation of NLRP3 inflammasome to relief symptom [27]. Another research showed that using A20 to block the NF-kB pathway in the joints reduced both the inflammatory response and the tissue destruction in CIA mice [44]. These researches indicated that A20 was a potential target for the treatment of RA. One of the active constituents of PZH, Ginsenoside $\mathrm{F} 1$, has been reported to have a regulatory effect on the expression of A20 [45]. In this study, we found that the protein level of A20 was significantly decreased in the joint of CIA mice when compared with the normal mice, which was consistent with the previous study. PZH could significantly increase the expression of A20 in the joints of CIA mice. It implied that the regulatory effect of PZH on NLRP3 inflammasome and NF- $\kappa B$ signaling pathway might be related to promoting the expression of A20.

In this study, the efficacy of PZH on CIA mice was evaluated, and the regulation of PZH on NF- $\mathrm{KB}$ signaling pathway and NLRP3 inflammasome was studied. However, the research on the effect and related mechanism of PZH on bone destruction in CIA mice was lacked in this study, although alleviation of bone destruction was found by histological analysis. In addition, previous studies indicated that A20 could also alleviate bone damage in CIA mice [44]. Therefore, we will focus on A20 and its related signal pathway to further investigate the effect of $\mathrm{PZH}$ on bone destruction in CIA mice.

\section{Conclusion}

In conclusion, this study preliminarily explored the effect and the possible action mechanism of $\mathrm{PZH}$ on CIA mice. The results showed that PZH performed a good therapeutic effect on CIA mice, and the mechanism might be related to the regulation of NF- $\kappa B$ signaling pathway and NLRP3 inflammasome.

\section{Abbreviations \\ RA: Rheumatoid arthritis; NLRP3: NOD-like receptor family pyrin domain- containing 3; NSAIDs: Non-steroidal anti-inflammatory drugs; TCM: Traditional Chinese medicines; PZH: Pien Tze Huang; CIA: Collagen-induced arthritis; MTX: Methotrexate.}

\section{Acknowledgements}

Not applicable.

\section{Authors' contributions}

YD performed the major research. HL and HS collected samples. JS, CL and NZ provided the technical support. YG and XH designed the study and revised the manuscript. AL supervised the whole study. All authors read and approved the final manuscript.

\section{Funding}

This research is supported by the National Key R\&D Program of China (2018YFC1705205).

\section{Availability of data and materials}

The datasets used and analyzed during the current study are available from the corresponding author on reasonable request.

\section{Ethics approval and consent to participate}

This study was reviewed and approved by the Research Ethics Committee of Institute of Basic Theory of Chinese Medicine, China Academy of Chinese Medical Sciences.

\section{Consent for publication}

Not applicable.

\section{Competing interests}

The authors declare that there are no competing interests.

\section{Author details}

1 Institute of Basic Research in Clinical Medicine, China Academy of Chinese Medical Sciences, Beijing, China. ${ }^{2}$ School of Life Science and Engineering, Southwest Jiaotong University, Chengdu, China. ${ }^{3}$ Institute of Clinical Medical Science, China-Japan Friendship Hospital, Beijing, China. ${ }^{4}$ The Second Clinical College of Guangzhou, University of Chinese Medicine, Guangzhou, China.

${ }^{5}$ Law Sau Fai Institute for Advancing Translational Medicine in Bone and Joint Diseases, School of Chinese Medicine, Hong Kong Baptist University, Kowloon Tong, Hong Kong, China. ${ }^{6}$ Academy of Integrative Medicine, Shanghai University of Traditional Chinese Medicine, Shanghai, China.

Received: 28 November 2019 Accepted: 17 March 2020

Published online: 30 March 2020

\section{References}

1. Nogueira E, et al. Update on therapeutic approaches for rheumatoid arthritis. Curr Med Chem. 2016;23(21):2190-203.

2. Brzustewicz E, Bryl E. The role of cytokines in the pathogenesis of rheumatoid arthritis-practical and potential application of cytokines 
as biomarkers and targets of personalized therapy. Cytokine. 2015;76(2):527-36.

3. Alghasham A, Rasheed Z. Therapeutic targets for rheumatoid arthritis: progress and promises. Autoimmunity. 2014;47(2):77-94.

4. Guo C, et al. NLRP3 inflammasome activation contributes to the pathogenesis of rheumatoid arthritis. Clin Exp Immunol. 2018;194(2):231-43.

5. Jue DM, Jeon Kl, Jeong JY. Nuclear factor kappaB (NF-kappaB) pathway as a therapeutic target in rheumatoid arthritis. J Korean Med Sci. 1999;14(3):231-8.

6. Shen HH, et al. NLRP3: a promising therapeutic target for autoimmune diseases. Autoimmun Rev. 2018;17(7):694-702.

7. Noort AR, Tak PP, Tas SW. Non-canonical NF-kappaB signaling in rheumatoid arthritis: Dr Jekyll and Mr Hyde? Arthritis Res Ther. 2015;17(1):15.

8. Burmester GR, Pope JE. Novel treatment strategies in rheumatoid arthritis. Lancet. 2017;389(10086):2338-48.

9. Guo Q, et al. Wu-Tou decoction in rheumatoid arthritis: integrating network pharmacology and in vivo pharmacological evaluation. Front Pharmacol. 2017;8(230):1-13.

10. Zhao H, et al. Yi Shen Juan Bi Pill ameliorates bone loss and destruction induced by arthritis through modulating the balance of cytokines released by different subpopulations of T cells. Front Pharmacol. 2018;9(262):1-11.

11. Qiu X, et al. Therapeutic potential of Pien Tze Huang on experimental autoimmune encephalomyelitis rat. J Immunol Res. 2018;2018(5):1-10.

12. Qiu X, et al. Pien Tze Huang alleviates relapsing-remitting experimental autoimmune encephalomyelitis mice by regulating Th1 and Th17 cells. Front Pharmacol. 2018;9(1237):1-13.

13. Kim HA, et al. Anti-arthritic effect of ginsenoside Rb1 on collagen induced arthritis in mice. Int Immunopharmacol. 2007;7(10):1286-91.

14. Zhang L, et al. Ginsenoside Rg1 attenuates adjuvant-induced arthritis in rats via modulation of PPAR-gamma/NF-kappaB signal pathway. Oncotarget. 2017;8(33):55384-93.

15. Song $W$, et al. Protective effect of ginsenoside metabolite compound $K$ against diabetic nephropathy by inhibiting NLRP3 inflammasome activation and NF-kappaB/p38 signaling pathway in high-fat diet/streptozotocin-induced diabetic mice. Int Immunopharmacol. 2018;63(1):227-38

16. Tsuji F, et al. Point mutation of tyrosine 759 of the IL-6 family cytokine receptor, gp130, augments collagen-induced arthritis in DBA/1J mice. BMC Musculoskelet Disord. 2009;10(23):1-10.

17. Zhang $L$, et al. Comparative efficacy of TACl-lg with TNF-alpha inhibitor and methotrexate in DBA/1 mice with collagen-induced arthritis. Eur J Pharmacol. 2013;708(1-3):113-23.

18. Liu $L$, et al. Acacetin regulated the reciprocal differentiation of Th17 cells and Treg cells and mitigated the symptoms of collagen-induced arthritis in mice. Scand J Immunol. 2018;88(4):e12712.

19. Lorenzo N, et al. APL-1, an altered peptide ligand derived from heatshock protein, alone or combined with methotrexate attenuates murine collagen-induced arthritis. Clin Exp Med. 2017;17(2):209-16.

20. Dong $R$, et al. Dataset on inflammatory proteins expressions and sialic acid levels in apolipoprotein E-deficient mice with administration of $\mathrm{N}$-acetylneuraminic acid and/or quercetin. Data Brief. 2016;8(11):613-7.

21. Benedetti G, Miossec P. Interleukin 17 contributes to the chronicity of inflammatory diseases such as rheumatoid arthritis. Eur I Immunol. 2014;44(2):339-47.

22. Yoshida Y, Tanaka T. Interleukin 6 and rheumatoid arthritis. Biomed Res Int. 2014;2014(23):1-14.

23. Thompson C, Davies R, Choy E. Anti cytokine therapy in chronic inflammatory arthritis. Cytokine. 2016;86(7):92-9.

24. Schiff $\mathrm{MH}$, et al. The safety of anakinra in high-risk patients with active rheumatoid arthritis: 6-month observations of patients with comorbid conditions. Arthritis Rheum. 2004;50(6):1752-60.

25. Scott LJ. Tocilizumab: a review in rheumatoid arthritis. Drugs. 2017;77(17):1865-79.
26. Devauchelle-Pensec V, Guellec D. Rheumatoid arthritis. Rev Prat. 2015;65(5):719-29.

27. Vande Walle L, et al. Negative regulation of the NLRP3 inflammasome by A20 protects against arthritis. Nature. 2014;512(7512):69-73.

28. van Loo G, Beyaert R. Negative regulation of NF-kappaB and its involvement in rheumatoid arthritis. Arthritis Res Ther. 2011;13(3):221.

29. Aravilli RK, Vikram SL, Kohila V. Phytochemicals as potential antidotes for targeting NF-kappaB in rheumatoid arthritis. 3 Biotech. 2017;7(4):253.

30. Messemaker TC, Huizinga TW, Kurreeman F. Immunogenetics of rheumatoid arthritis: understanding functional implications. J Autoimmun. 2015;64(8):74-81.

31. Kim DH, et al. Ginsenoside Rd inhibits the expressions of iNOS and COX-2 by suppressing NF-kappaB in LPS-stimulated RAW264.7 cells and mouse liver. J Ginseng Res. 2013;37(1):54-63.

32. Lian LH, et al. Ginsenoside Rh2 downregulates LPS-Induced NF-kappa $B$ activation through inhibition of TAK1 phosphorylation in RAW 264.7 murine macrophage. Evid Based Complement Alternat Med. 2013;2013(28):1-14

33. Ahn S, et al. Suppression of MAPKs/NF-kappaB activation induces intestinal anti-inflammatory action of ginsenoside Rf in HT-29 and RAW264.7 cells. Immunol Invest. 2016;45(5):439-49.

34. Xia KP, Ca HM, Shao CZ. Protective effect of notoginsenoside R1 in a rat model of myocardial ischemia reperfusion injury by regulation of Vitamin D3 upregulated protein 1/NF-kappaB pathway. Pharmazie. 2015;70(11):740-4.

35. Jin C, Flavell RA. Molecular mechanism of NLRP3 inflammasome activation. J Clin Immunol. 2010;30(5):628-31.

36. Zhao C, et al. NLRP3 inflammasome regulates Th17 differentiation in rheumatoid arthritis. Clin Immunol. 2018;197(9):154-60.

37. Wang T, et al. Role of NLRP3 and NLRP1 inflammasomes signaling pathways in pathogenesis of rheumatoid arthritis. Asian Pac J Trop Med. 2014;7(10):827-31.

38. Du J, et al. Ginsenoside Rg1, a novel glucocorticoid receptor agonist of plant origin, maintains glucocorticoid efficacy with reduced side effects. J Immunol. 2011;187(2):942-50.

39. Vereecke L, Beyaert R, van Loo G. Genetic relationships between A20/ TNFAIP3, chronic inflammation and autoimmune disease. Biochem Soc Trans. 2011;39(4):1086-91.

40. Matsumoto I, et al. Regulatory roles of tumor necrosis factor alphainduced proteins (TNFAIPS) 3 and 9 in arthritis. Clin Immunol. 2014;153(1):73-8.

41. Elsby LM, et al. Functional evaluation of TNFAIP3 (A20) in rheumatoid arthritis. Clin Exp Rheumatol. 2010;28(5):708-14.

42. Zhou Q, Wang H, Schwartz DM. Loss-of-function mutations in TNFAIP3 leading to A20 haploinsufficiency cause an early-onset autoinflammatory disease. Nat Genet. 2016;48(1):67-73.

43. Wang Z, et al. Altered TNFAIP3 mRNA expression in peripheral blood mononuclear cells from patients with rheumatoid arthritis. Biomed Rep. 2015;3(5):675-80.

44. Hah YS, et al. A20 suppresses inflammatory responses and bone destruction in human fibroblast-like synoviocytes and in mice with collageninduced arthritis. Arthritis Rheum. 2010;62(8):2313-21.

45. Qin M, et al. Ginsenoside F1 ameliorates endothelial cell inflammatory injury and prevents atherosclerosis in mice through A20-mediated suppression of NF-kB signaling. Front Pharmacol. 2017;8(11):953-65.

\section{Publisher's Note}

Springer Nature remains neutral with regard to jurisdictional claims in published maps and institutional affiliations. 